\title{
Granulocyte-Macrophage Colony-Stimulating Factor Production and Tissue Eosinophilia in Chronic Rhinitis
}

\author{
Aleksandar Peric ${ }^{1} \quad$ Cveta Spadijer-Mirkovic $^{1} \quad$ Svjetlana Matkovic-Jozin ${ }^{2} \quad$ Ljiljana Jovancevic $^{3}$ \\ Danilo Vojvodic ${ }^{4}$
}

${ }^{1}$ Department of Otorhinolaryngology, Military Medical Academy, Belgrade, Serbia

${ }^{2}$ Department of Otorhinolaryngology, Stavanger University Hospital, Stavanger, Norway

${ }^{3}$ Department of Otorhinolaryngology, Clinical Center of Vojvodina, Novi Sad, Serbia

${ }^{4}$ Institute of Medical Research, Division of Clinical and Experimental Immunology, Military Medical Academy, Belgrade, Serbia

\begin{abstract}
Address for correspondence Aleksandar Peric, MD, PhD, Department of Otorhinolaryngology, Military Medical Academy, Crnotravska 17, Belgrade, Belgrade 11000, Serbia (e-mail: alexneta@orion.rs).
\end{abstract}

Int Arch Otorhinolaryngol 2016;20:364-369.

\begin{abstract}
Keywords

- rhinitis

- allergic

- perennial

- nasal lavage fluid

- eosinophilia

- cytokines

Introduction Granulocyte-macrophage colony-stimulating factor (GM-CSF) is a strong proinflammatory cytokine that takes part in allergic nasal inflammation as an eosinophil colony-stimulating factor. However, the role of GM-CSF in non-allergic rhinitis has not been fully explored.

Objectives The aim of this investigation was to assess the concentration of GM-CSF in nasal secretions of patients with non-allergic rhinitis with eosinophilia syndrome (NARES) in comparison to patients with perennial allergic rhinitis (PAR) and healthy subjects, as well as to assess the relationship with the degree of eosinophilic inflammation and clinical characteristics of the patients.

Methods Fourteen patients with diagnosis of NARES, 14 PAR patients, and 14 healthy subjects were included in this cross-sectional study. All patients underwent symptom score assessment, nasal endoscopy, allergy testing, and cytological evaluation. The concentration of GM-CSF in nasal secretions of all participants was measured by enzyme-linked immunosorbent assay (ELISA).

Results We found significantly higher levels of GM-CSF in patients with NARES than in the control group $(p=0.035)$. The percent of eosinophils in nasal mucosa was higher in NARES patients in comparison to patients with PAR $(p<0.001)$ and control patients $(p<0.0001)$. We found positive correlations between GM-CSF levels and eosinophil counts only in NARES patients.

Conclusion The concentrations of GM-CSF in nasal secretions correlate well with eosinophil counts in the nasal mucosa of NARES patients. These facts indicate a possible role of GM-CSF as a favorable marker for assessment of nasal disease severity and the degree of chronic eosinophilic inflammation in the nasal mucosa.
\end{abstract}

received

October 19, 2015

accepted

November 6, 2015

published online

February 26, 2016
DOI http://dx.doi.org/

$10.1055 / \mathrm{s}-0035-1570746$ ISSN $1809-9777$.
Copyright $(2016$ by Thieme Publicações License terms

Ltda, Rio de Janeiro, Brazil
$(\Theta(1) \Theta \circledast$ 


\section{Introduction}

Unlike allergic rhinitis (AR), there are no specific diagnostic tests for non-allergic rhinitis (NAR). Diagnosis is primarily based on rhinitis symptoms, which include nasal congestion, rhinorrhea, sneezing, itching, and impaired sense of smell, for greater than one hour most days in the absence of identifiable allergy by allergy testing. ${ }^{1}$ AR is an immunoglobulin $\mathrm{E}$ (IgE)-mediated noninfectious disease of the nasal mucosa following contact with allergens. Previous studies have demonstrated that imbalance of T helper 1 / T helper 2 (Th1/Th2) cell-mediated immunity plays an important role in the pathogenesis of AR, which is characterized by the Th2 cell mediated inflammation. ${ }^{2}$ Although chronic inflammation has proven to be an integral component of $A R$, there is great debate regarding this facet in NAR, since some studies have suggested that exclusion of inflammation is indicative in vasomotor rhinitis. Other studies have demonstrated that all patients with non-allergic rhinitis with eosinophilia syndrome (NARES) have high degree of chronic eosinophilic inflammation. ${ }^{3,4}$ NARES, which accounts for $\sim 14 \%$ of rhinitis patients, is defined by a syndrome of nasal hyper-reactivity for more than three months, the absence of atopic factor, and a profound nasal eosinophilia with more than $20 \%$ eosinophils in the total granulocytic or mononuclear cell population. ${ }^{4}$

It is well-known that many cytokines play a role in the manifestation of nasal allergic reaction through the activation and proliferation of migrating cells, such as eosinophils, mastocytes, and lymphocytes, as well as nasal mucosa epithelial cells. These cells produce a variety of cytokines that, in turn, regulate the immunological reaction and inflammatory process. ${ }^{5}$ Granulocyte-macrophage colony-stimulating factor (GM-CSF) is a hematopoietic growth factor, which was originally recognized as a stimulator of the proliferation of granulocytes and macrophages from bone marrow precursor cells. ${ }^{6}$ The main sources of GM-CSF in allergic rhinitis include epithelial and endothelial cells, activated eosinophils, T and B cells, monocytes, and macrophages. ${ }^{6,7}$ GM-CSF also reportedly takes part in Th2 response in allergic nasal inflammation as an eosinophil colony-stimulating factor and by activation of dendritic cells. ${ }^{7}$ On the other hand, in non-allergic and aspirin tolerant patients with chronic polypous rhinosinusitis, eosinophils appear to be recruited mainly by the release of GM-CSF. ${ }^{8}$ However, the role of GM-CSF in pathogenesis of NARES has not been fully explored.

The aim of this investigation was to assess the concentration of GM-CSF in nasal secretions in patients with NARES in comparison to patients with perennial allergic rhinitis (PAR) and healthy subjects, and to assess the relationship with the degree of eosinophilic inflammation and clinical characteristics of these patients.

\section{Materials and Methods}

\section{Participants}

We recruited 14 patients with diagnosis of NARES ( 9 men and 5 women, mean age $42.38 \pm 11.18$ years) and 14 patients with diagnosis of PAR ( 8 men and 6 women, mean age $41.05 \pm 9.78$ years) for participation in this cross-sectional study, which was performed in accordance with the Declaration of Helsinki. The protocol and methods received approval from our institutiońs Ethics Committee. We obtained written informed consent from all patients. This study was performed in the Rhinology Unit of the Department of Otorhinolaryngology between May 2013 and April 2015. As controls in the study, we included fourteen healthy subjects without symptoms, medical history, or endoscopic findings of nasal/paranasal sinus inflammation. The main age in the control group ( 7 male and 7 female subjects) was $40.58 \pm 13.37$ years.

Following the Allergic Rhinitis and its Impact on Asthma (ARIA) guidelines, ${ }^{9}$ we divided patients with AR into two categories: intermittent and persistent. We only included patients with persistent symptoms (more than 4 days a week and for more than 4 weeks) in the study to avoid differences due to actual allergen exposure between seasonal and non-seasonal subjects. The patients with PAR had typical nasal symptoms (rhinorrhea, sneezing, itching, nasal obstruction, hyposmia) for at least 12 weeks. They had the confirmation of atopic status, negative nasal endoscopy for polyps, and negative computed tomography (CT) scan of paranasal sinuses for mucosal swelling. The patients with NARES complained about typical symptoms of PAR (rhinorrhea, sneezing, itching, nasal obstruction, and hyposmia) for more than 12 weeks. However, all allergy tests were negative for atopy. Nasal hypereosinophilia was found by scraping of nasal mucosa of the inferior turbinate and more than $20 \%$ eosinophils in the total granulocyte and mononuclear cell population, excluding respiratory epithelium cells, was the criteria for a NARES diagnosis. CT scan was negative in all subjects. We evaluated the presence of micropolyposis by nasal endoscopy. An endoscopic finding was understood as characteristic of chronic rhinosinusitis (CRS) and we excluded such patients for further investigation.

Exclusion criteria were: chronic polypous rhinosinusitis (including endoscopic evidence of micropolyposis), bronchial asthma, systemic diseases affecting the nose (sarcoidosis, primary ciliary dyskinesia, Wegener's granulomatosis, cystic fibrosis, Churg-Strauss syndrome). Also, the patients with a history of cigarette smoking and previous nasal and paranasal sinus surgery were excluded. None of the patients had any acute upper and lower respiratory tract infections, use of antibiotics, oral or intranasal antihistamines, and systemic or topical corticosteroids within three weeks before the start of this investigation.

\section{Allergy Determination}

The atopic status was evaluated in all participants at the start of the study by an allergist on the basis of clinical symptoms, medical history of allergic rhinitis, positive skin-prick tests, and positive serological test. Skin-prick tests were performed on the volar part of the forearm with a standard battery of common aeroallergens including house dust mite (Dermatophagoides farinae, Dermatophagoides pteronyssinus), fungus (Alternaria alternata, Aspergillus fumigatus), dogs, and cats. We also included negative ( $0.9 \%$ natrium-chloridum solution) and positive ( $1 \mathrm{mg} / \mathrm{ml}$ histamine dihydrochloride solution) controls with each skin-prick tests. After 15 minutes, we read 
the reactions. We considered the test positive if the diameter of wheal was greater than $3 \mathrm{~mm}$ with respect to the negative control. We measured total serum IgE level by enzyme-linked immunosorbent assay (ELISA) kit (Elitech Diagnostics, Salonde-Provence, France) and an ELISA reader (Spectra III, Austria). We then collected venous blood and centrifuged it, and stored the serum at $-70^{\circ} \mathrm{C}$ until testing. Subjects were considered allergic if they had a serum IgE level $>100 \mathrm{IU} / \mathrm{mL}$.

\section{Symptoms}

The same rhinologist examined all the patients. The examiner asked all NARES and PAR patients to assess their symptoms (nasal obstruction, rhinorrhoea, hyposmia, sneezing, and itching). The symptoms were scored from 0 to 3: 0 for no symptoms, 1 for mild symptoms, 2 for moderate symptoms, and 3 for severe symptoms, resulting in a maximum nasal symptom score of 15 , as previously described. ${ }^{10}$

\section{Nasal Cytology}

We counted the number of granulocytes on nasal scraped tissue obtained from the inferior turbinate bilaterally by rhinoprobe. The cupped tip of the disposable probe was gently passed over the mucosal surface. Two or three short scrapes of the epithelial layer are made to obtain a sample. The specimen was spread onto a plain slide and immediately fixed for at least one minute in 95\% ethyl alcohol and stained with May Grünwald-Giemsa. An experienced cytologist blindly examined the samples, unaware of the clinical status of participants. We counted the percentage of eosinophils by microscopic cytological examination. The slides were examined under oil immersion by light microscopy at a magnification of $\times 400$. We expressed eosinophil counts as a percentage of cells of the granulocytic or mononuclear type, without nasal epithelial cells, per high-power field, from a mean of at least 10 fields observed.

\section{Sampling of Nasal Secretions and GM-CSF Determination}

We collected nasal secretion samples from nasal cavities of all 42 subjects, 14 with PAR, 14 with NARES, and 14 healthy subjects, using the absorption technique. We used cottonwool sticks (length $10 \mathrm{~mm}$, diameter $4 \mathrm{~mm}$; Institute of Virology, Vaccines and Sera, Torlak, Belgrade, Serbia). We inserted them for 5 minutes into the middle meatus, under the endoscopic guidance, as previously described. ${ }^{11,12} \mathrm{We}$ placed all samples in a $2 \mathrm{~mL}$ Eppendorf tube containing $1 \mathrm{~mL}$ of transfer medium (phosphate-buffered saline with gentamicin $50 \mu \mathrm{g} / \mathrm{mL}$, penicillin $\mathrm{G} 340 \mathrm{IU} / \mathrm{mL}$, fungizone $500 \mu \mathrm{g} / \mathrm{mL}$ ) for 30 minutes. It allowed the diffusion of cytokines into the medium and then stored at $4^{\circ} \mathrm{C}$ for a maximum of 2 hours until processed. We centrifuged nasal fluid at $1000 \mathrm{~g}$ for 10 minutes to separate the cellular components. After centrifugation, we portioned supernatants and stored at $-70^{\circ} \mathrm{C}$ for no more than two months, pending cytokine determination. We measured levels of GM-CSF in all of the 42 samples using the commercial human ELISA kit (Thermo Fisher Scientific Inc., Waltham, MA, U.S.A.). We expressed the concentrations of GM-CSF in picograms per milliliter $(\mathrm{pg} / \mathrm{mL})$. The sensitivity of detection was $<2 \mathrm{pg} / \mathrm{mL}$ and assay ranged from $15.4 \mathrm{pg} /$ $\mathrm{mL}$ to $600 \mathrm{pg} / \mathrm{mL}$. According to the producer's declaration, overall intra-assay and inter-assay coefficient of variation should not exceed $10 \%$.

\section{Statistical Analysis}

We expressed data as mean \pm standard deviation (SD). We analyzed comparisons between the groups using the nonparametric Mann-Whitney U-test. We explored the strength of the correlation between different parameters using the Spearman's rank correlation test. P values $<0.05$ were considered significant. We performed the analysis using the SPSS software (Statistical Package for the Social Sciences, version 15.0, SPSS Inc., Chicago, U.S.A.).

\section{Results}

\section{Patients' Characteristics}

A statistically significant difference between NARES and PAR patients was found in the nasal symptom score $(p=0.047)$. On the other hand, we found significantly higher concentrations of total serum IgE in patients with PAR than in patients with NARES $(148.98 \pm 67.25 \mathrm{IU} / \mathrm{mL}$ versus $87.35 \pm 27.48 \mathrm{IU} / \mathrm{mL})(p=0.029)$. The patients' characteristics are presented in -Table $\mathbf{1}$.

\section{GM-CSF Levels and Eosinophil Counts}

We detected GM-CSF in 13 of 14 nasal secretion samples of patients with NARES. However, we could not detect such cytokine in samples of 5 patients with PAR and 6 controls. The mean concentration of GM-CSF in nasal secretions was significantly higher in NARES patients $(33.01 \pm 23.45 \mathrm{pg} / \mathrm{mL}$ ) compared with control patients $(21.35 \pm 23.28 \mathrm{pg} / \mathrm{mL})(p=0.035)$. We observed no significant difference in GM-CSF levels between PAR patients $(26.08 \pm 27.92 \mathrm{pg} / \mathrm{mL})$ and patients with diagnosis of NARES ( $p=0.064)$. We also found no significant difference in the GM-CSF concentration between PAR patients and healthy subjects $(p=0.127)$ (-Fig. 1).

The mean eosinophil percentage observed in the PAR patients, NARES patients, and control patients were $27.88 \pm 8.73,51.85 \pm 11.82$, and $5.92 \pm 2.97$, respectively. The highest eosinophil count was found in the patients with NARES with significant differences compared with PAR patients $(p<0.001)$ and control patients $(p<0.0001)$.

Table 1 Patients' characteristics

\begin{tabular}{|l|l|l|}
\hline & NARES & PAR \\
\hline Number of patients & 14 & 14 \\
\hline Age/Years* & $42.38 \pm 11.18$ & $41.05 \pm 9.78$ \\
\hline Men/Women & $9 / 5$ & $8 / 6$ \\
\hline Nasal symptom score & $11.45 \pm 2.37$ & $8.47 \pm 1.95$ \\
\hline Total serum IgE (IU/ml)* $^{*} 87.35 \pm 27.48$ & $148.98 \pm 67.25$ \\
\hline
\end{tabular}

Abbreviations: NARES, non-allergic rhinitis with eosinophilia syndrome; PAR, perennial allergic rhinitis; IgE, immunoglobulin $E$.

*Mean \pm standard deviation. 


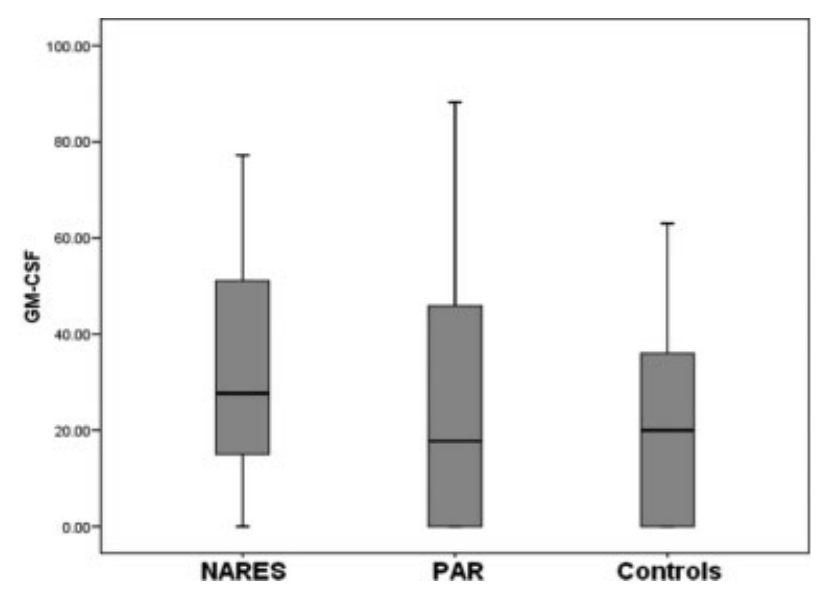

Fig. 1 Concentrations of GM-CSF in nasal secretions of patients with non-allergic rhinitis with eosinophilia syndrome (NARES), perennial allergic rhinitis (PAR), and in healthy subjects.

Therefore, PAR patients have higher eosinophil count in the nasal mucosa than healthy subjects $(p<0.001)$.

We only found a significant positive correlation between GM-CSF levels in nasal secretions and eosinophil counts in the nasal mucosa $(r=0.552, p=0.01)$ in patients with NARES (-Fig. 2). There were no significant correlations found between GM-CSF concentrations in nasal secretions and symptoms/eosinophil percentage in patients with PAR (-Table 2). In control subjects, we also found no correlation between GM-CSF levels and eosinophil counts.

\section{Discussion}

Nasal secretions are a mixture of plasma exudation and mucus produced by goblet cells and seromucous glands, together with plenty of epithelial and migrating cells such as granulocytes, lymphocytes, and mononuclear cells with immunocompetent activities. The biochemical and cytological exploration of nasal secretions may provide additional information on mucosal activity. ${ }^{13}$ Studies have shown that contents of nasal secretions reflect the inflammatory status of

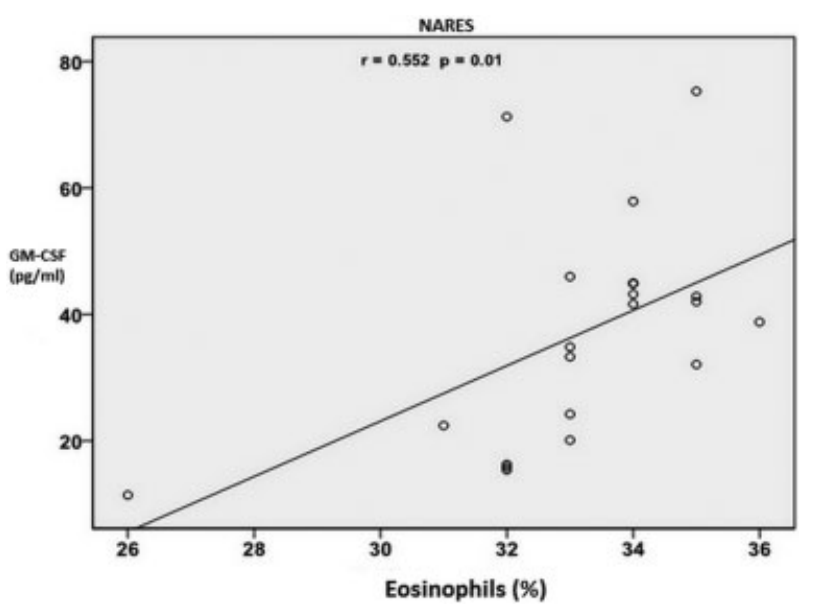

Fig. 2 Correlation between GM-CSF levels in nasal secretions and eosinophil counts in nasal mucosa was found only in NARES patients.
Table 2 Correlations

\begin{tabular}{|l|l|l|}
\hline $\begin{array}{l}\text { GM-CSF } \\
\text { concentration }\end{array}$ & $\begin{array}{l}\text { Nasal symptom } \\
\text { score }\end{array}$ & $\begin{array}{l}\text { Eosinophil } \\
\text { counts }\end{array}$ \\
\hline NARES & $\mathrm{R}=0.318$ & $\mathrm{R}=0.552^{*}$ \\
& $p=0.084$ & $p=0.01$ \\
\hline PAR & $\mathrm{R}=0.327$ & $\mathrm{R}=0.348$ \\
& $p=0.089$ & $p=0.069$ \\
\hline
\end{tabular}

Abbreviations: GM-CSF, Granulocyte-Macrophage Colony-Stimulating Factor; NARES, non-allergic rhinitis with eosinophilia syndrome; PAR, perennial allergic rhinitis.

${ }^{*}$ Correlation is significant at the 0.01 level (2-tailed).

the nasal mucosa, paralleling the evolution of mucosal disease. ${ }^{14}$ Previous investigations showed that cytokine and chemokine levels in nasal secretions correlate well with clinical parameters and cytological findings in patients with chronic upper airway inflammatory diseases. ${ }^{11,12,15}$ On the other hand, cytological examination of the nasal secretions and nasal mucosa is a helpful path towards better knowledge of the pathophysiology of chronic inflammatory diseases and correct differential diagnosis. $^{16,17}$

NARES is a chronic inflammatory disease of unknown origin. It is characterized by nasal symptoms consistent with allergic rhinitis in which an absence of atopy has been demonstrated by allergen skin testing and serological testing. The pathophysiology of NARES is poorly understood, but a key component involves a self-perpetuating, chronic eosinophilic nasal inflammation with development of nasal micropolyposis during the transformation in chronic polypous rhinosinusitis. ${ }^{4}$ The high level of release of substance $P$ in the nasal mucosa lead to the hypothesis of a neurogenic origin of NARES. ${ }^{18}$ This disease is a risk factor for the development of nasal polyposis associated with aspirin sensitivity. Treatment consists mainly of intranasal corticosteroid drops and sprays, with or without the addition of oral second-generation antihistamines and/or leukotriene-receptor antagonists. ${ }^{1,4}$ Our results showed significantly higher production of cytokine GM-CSF in the nasal mucosa of patients with NARES than in the control group. The level of eosinophilic infiltration of the nasal mucosa in NARES patients is two times higher than in the patients with PAR and almost ten times higher than in the control patients. Ohkubo et $\mathrm{al}^{19}$ demonstrated that epithelial cells are a main source of CM-CSF in nasal secretions of healthy subjects, whereas in patients with allergic rhinitis, the main sources are migrating cells (eosinophils and lymohocytes) and epithelial cells, induced by antigen stimulation.

In our results, for NARES patients, GM-CSF concentration in nasal fluid correlates well with eosinophil counts in the nasal mucosa. This finding suggests that the main source of the cytokine in patients with NARES are activated eosinophils. The fact that basal secretions of GM-CSF were greater in NARES patients than in the patients with PAR (although without significant difference) may explain the higher abundance of eosinophils in the nasal mucosa of NARES patients. This proinflammatory cytokine may influence the growth, differentiation, proliferation, and activation of eosinophils, 
which explain the good correlation between GM-CSF levels in nasal secretions and eosinophil counts in our results. The results of a previous study showed that GM-CSF is the main cytokine in the process of eosinophil activation in chronic rhinosinusitis with nasal polyps (CRSwNP). ${ }^{20}$ Moreover, the receptor affinity of GM-CSF is almost 10 times stronger than that of interleukin-3 (IL-3) or IL-5. ${ }^{20}$ Many facts indicate that chronic eosinophilic inflammation in patients with NARES have similar characteristics to those found in CRSwNP. According to the results presented by Moneret-Vautrin et $a{ }^{18}{ }^{18}$ NARES seems to evolve in three stages: (1) migration of eosinophils from the vessels of the nasal mucosa to the nasal secretions; (2) retention of eosinophils in the mucosa, which might be linked to activation of unknown origin; and (3) nasal polyposis. However, relatively frequent association with aspirin sensitivity implies that this disease should be understood as a distinct entity among the different types of chronic sinonasal inflammations.

In our review of the literature, we found only one recently published study concerning the association between GM-CSF levels in nasal secretions and nasal eosinophilia. De Corso et $\mathrm{al}^{21}$ found detectable levels of this cytokine in 34 of 70 (48.57\%) patients, with an average concentration of $2.67 \pm$ $0.8 \mathrm{pg} / \mathrm{ml}$, whereas, only 1 out of 20 individuals in the control group showed detectable GM-CSF levels. In our study, we found detectable levels of GM-CSF in 22 of 28 patients (71.57\%). We could not detect cytokine levels in only 6 control subjects. Different methods for nasal secretions sampling could explain the differences between our study and that of De Corso et al regarding the detectability of GM-CSF and average cytokine concentrations. De Corso et $\mathrm{al}^{21}$ performed the nasal lavage dilution technique, whereas we used the absorption technique to collect nasal secretions. In the dilution technique, a liquid is instilled into the nose, recovered with an admixed and sample of epithelial lining fluid. Thus, nasal lavage is associated with a substantial, often unpredictable, dilution of nasal secretions. As a consequence, the concentration of inflammatory mediators may reveal high variability and frequently falls below the lower detection limits. On the other hand, the absorption technique overcomes the problem encountered when only small quantities of spontaneous secretions are available, as it provides sufficient amounts of undiluted nasal secretions. Riechelmann et $\mathrm{al}^{22}$ for instance, found that analyte concentrations in nasal lavage were $\sim 10$ times lower than in specimens obtained by the absorption technique.

\section{Conclusions}

Our results demonstrated that proinflammatory cytokine GM-CSF production and eosinophilic inflammation are higher in patients with NARES than in the patients with PAR and in healthy subjects. The concentrations of this cytokine in nasal secretions correlate well with eosinophils counts in the nasal mucosa only in NARES patients. The measurement of local inflammatory mediators in nasal secretions could be a useful path in the monitoring of the severity of chronic nasal inflammation, as well as a sensitive way to study the pathogenesis of these diseases. Our results indicate a possible role of GM-CSF as a favorable marker for the investigation of pathophysiological mechanisms, which play a role in the development of NARES. Nonetheless, further studies in this direction conducted with a higher number of patients are needed.

\section{Conflict of Interest Statement}

The authors declare that they have no conflict of interest regarding the publication of this paper.

\section{References}

1 Papadopoulos NG, Bernstein JA, Demoly P, et al. Phenotypes and endotypes of rhinitis and their impact on management: a PRACTALL report. Allergy 2015;70(5):474-494

2 Huang X, Chen Y, Zhang F, Yang Q, Zhang G. Peripheral Th17/Treg cell-mediated immunity imbalance in allergic rhinitis patients. Braz J Otorhinolaryngol 2014;80(2):152-155

3 Nguyen KH, Suzuki H, Wakasugi T, et al. Expression of epidermal growth factors and a tight junction protein in the nasal mucosa of patients with chronic hypertrophic rhinitis. Allergol Immunopathol (Madr) 2013;41(4):246-254

4 Ellis AK, Keith PK. Nonallergic rhinitis with eosinophilia syndrome. Curr Allergy Asthma Rep 2006;6(3):215-220

5 Scavuzzo MC, Rocchi V, Fattori B, et al. Cytokine secretion in nasal mucus of normal subjects and patients with allergic rhinitis. Biomed Pharmacother 2003;57(8):366-371

6 Shiomi A, Usui T. Pivotal roles of GM-CSF in autoimmunity and inflammation. Mediators Inflamm 2015;2015:568543

7 Shiozawa A, Miwa M, Ono N, Homma H, Hirotsu M, Ikeda K. Comparative analysis of cytokine release from epithelial cell cultures of the upper airway. Rhinology 2015;53(2):135-141

8 Rinia AB, Kostamo K, Ebbens FA, van Drunen CM, Fokkens WJ. Nasal polyposis: a cellular-based approach to answering questions. Allergy 2007;62(4):348-358

9 Van Hoecke H, Van Cauwenberge P, Thas O, Watelet JB. The ARIA guidelines in specialist practice: a nationwide survey. Rhinology 2010;48(1):28-34

10 Tsicopoulos A, Shimbara A, de Nadai P, et al. Involvement of IL-9 in the bronchial phenotype of patients with nasal polyposis. J Allergy Clin Immunol 2004;113(3):462-469

11 Perić A, Baletić N, Sotirović J, Špadijer-Mirković C. Macrophage inflammatory protein-1 production and eosinophil infiltration in chronic rhinosinusitis with nasal polyps. Ann Otol Rhinol Laryngol 2015;124(4):266-272

12 Perić A, Vojvodić D, Perić AV, Radulović V, Miljanović O. Correlation between cytokine levels in nasal fluid and scored clinical parameters in patients with nasal polyposis. Indian J Otolaryngol Head Neck Surg 2013;65(Suppl 2):295-300

13 Watelet JB, Gevaert P, Holtappels G, Van Cauwenberge P, Bachert C. Collection of nasal secretions for immunological analysis. Eur Arch Otorhinolaryngol 2004;261(5):242-246

14 Lü FX, Esch RE. Novel nasal secretion collection method for the analysis of allergen specific antibodies and inflammatory biomarkers. J Immunol Methods 2010;356(1-2):6-17

15 De Corso E, Baroni S, Romitelli F, et al. Nasal lavage CCL24 levels correlate with eosinophils trafficking and symptoms in chronic sino-nasal eosinophilic inflammation. Rhinology 2011;49(2): 174-179

16 Jankowski R, Persoons M, Foliguet B, Coffinet L, Thomas C, Verient-Montaut B. Eosinophil count in nasal secretions of subjects with and without nasal symptoms. Rhinology 2000; 38(1):23-32 
17 de Corso E, Battista M, Pandolfini M, et al. Role of inflammation in non-allergic rhinitis. Rhinology 2014;52(2):142-149

18 Moneret-Vautrin DA, Jankowski R, Bene MC, et al. NARES: a model of inflammation caused by activated eosinophils? Rhinology 1992;30(3):161-168

19 Ohkubo K, Ikeda M, Pawankar R, Gotoh M, Yagi T, Okuda M. Mechanisms of IL-6, IL-8, and GM-CSF release in nasal secretions of allergic patients after nasal challenge. Rhinology 1998;36(4): 156-161
20 Shin SH, Lee SH, Jeong HS, Kita H. The effect of nasal polyp epithelial cells on eosinophil activation. Laryngoscope 2003;113(8):1374-1377

21 De Corso E, Baroni S, Lucidi D, et al. Nasal lavage levels of granulocyte-macrophage colony-stimulating factor and chronic nasal hypereosinophilia. Int Forum Allergy Rhinol 2015;5(6): 557-562

22 Riechelmann H, Deutschle T, Friemel E, Gross HJ, Bachem M. Biological markers in nasal secretions. Eur Respir J 2003;21(4): 600-605 